\title{
An Efficient Non-Uniform Multi-Tone System Based On Ramanujan Sums
}

\author{
Qinbiao Yang ${ }^{1}$, Zulin Wang ${ }^{1,2}$, Qin Huang ${ }^{1}$ \\ 1 Electronic and Information Engineering, Beihang University, Beijing, 100191, China \\ 2 Collaborative Innovation Center of Geospatial Technology, Wuhan, 43079, China
}

Keywords: Multi-carrier; Non-uniform; Ramanujan Sums; RFMT

\begin{abstract}
Non-uniform multi-carrier Ramanujan Fourier Multi-tone (RFMT) systems have low transmission efficiency because of their large transform length. This paper demonstrated that the signs of received symbols before demodulation remain unchanged as long as the product of transform matrix and its transpose is dominated by its diagonal elements. Thus, it is possible to recover the transmitted bits from truncated transform vectors. Analysis and simulation results show that the truncated RFMT realizes non-uniform spectrum distribution similar to that of RFMT. When its transmission efficiency is twice of that of RFMT, i.e. the transform length is truncated to half of the original length, it still has the same anti-noise performance as RFMT. Thus, the truncated RFMT systems not only enjoy higher transmission efficiency, but also enable a flexible tradeoff between efficiency and reliability for various channel qualities.
\end{abstract}

\section{Introduction}

Multi-tone modulation[1] is an efficient transmission scheme and effectively against the multipath fading effect. Orthogonal Frequency Division Multiplexing(OFDM)[2] is a classical multi-tone modulation system with uniform carrier distribution. It has been applied to the 4G mobile communication systems, Wi-Fi and some military communication systems. However, in the rapidly timevarying and frequency selectivity channel, the reliability of the uniform carrier distribution will decline seriously and even collapse. In order to solve this problem, the non-uniform carrier distribution systems are developed.

The non-uniform carrier distribution systems adjust the carrier distribution according to the channel state information(CSI)[3-5]. More carriers will be allocated to the high quality frequency bands and the worse bands will get less carriers or none. Therefore, the non-uniform scheme will obtain greater performance than the uniform in the dynamic channel. Ramanujan Sums(RS)[6,7] based RFMT systems[8,9] realize non-uniform carrier distribution with the transform matrix composed of RS set. They can adjust the carrier distribution according to CSI. They outperform uniform multi-carrier systems in dynamic channels. Moreover, their peak-average power rate(PAPR) and computational complexity are less than the other non-uniform schemes[10], including non-contiguous OFDM(NC-OFDM)[11,12] and Warped Discrete Fourier Transform(WDFT) based nonuniform multi-carrier system[13,14].

In RFMT, the size of transform matrix or transform vector may be very large since both the signs and amplitudes of the transmitted symbols are exactly recovered. Large transform length lowers the transmission efficiency. This paper reveals that only the signs of the received symbols are sufficient to recover the transmitted bits. Moreover, it demonstrates that the signs of the received symbols keep unchanged as long as the product of transform matrix and its transpose is dominated by its diagonal elements. In view of the above, a transform length truncated RFMT(TLT-RFMT) is proposed to achieve much better transmission efficiency with short transform vector. Moreover, the proposed TLT-RFMT not only enables a flexible tradeoff between efficiency and reliability for different channel qualities, but also enjoys lower complexity than RFMT.

The rest of this paper is organized as follows. Section 2 provides background on RFMT system. Section 3 constructs TLT-RFMT system and demonstrates the principle of the transform length truncated. Section 4 evaluates the proposed scheme TLT-RFMT through simulation. Finally, Section 5 concludes this paper.

\section{RFMT system}

The transform basis of RFMT system is based on RS, which are sums of the $n^{\text {th }}$ powers of $q^{\text {th }}$ primitive roots of unity[6],

$$
c_{q}(n)=\sum_{p=1}^{q} \exp \left(2 \mathrm{i} \pi n \frac{p}{q}\right),(p, q)=1
$$

where $q$ means the sequence number of the RS, $(p, q)=1$ means $p$ and $q$ are co-prime, and i means the imaginary unit. Define

$$
\mathbf{x}=\left(x_{1}, x_{2}, \cdots, x_{N}\right), x_{1}, x_{2}, \cdots, x_{N} \in\{+1,-1\}
$$

as the modulated symbols vector in the binary phase-shift keying(BPSK), and

$$
\mathbf{c}_{t}=\left(c_{t}(1), c_{t}(2), \cdots, c_{t}(L)\right)^{\mathrm{T}}, t \in\left\{q_{1}, q_{2}, \cdots, q_{N}\right\}
$$

as the transform vector. Then the transform matrix $\mathbf{R}_{\mathrm{s}}$ can be written

$$
\mathbf{R}_{\mathrm{s}}=\left[\mathbf{c}_{q_{1}}, \mathbf{c}_{q_{2}}, \cdots, \mathbf{c}_{q_{N}}\right]^{\mathrm{T}}
$$

where $L$ is the complete transform length and $\mathrm{T}$ means the transpose of the vector. In RFMT system, the inversion 
Ramanujan-Fourier Transform(IRFT) and Ramanujan-Fourier Transform(RFT)[15] of $\mathbf{x}$ are defined as follows,

$$
\begin{aligned}
& \mathbf{x}= \\
& \frac{1}{L}\left(X_{1}, X_{2}, \cdots, X_{L}, \cdots, X_{L}\right)=\mathbf{x} \cdot \mathbf{R}_{\mathrm{s}}^{\mathrm{T}} \operatorname{diag}\left(\frac{1}{\varphi\left(q_{1}\right)}, \frac{1}{\varphi\left(q_{2}\right)}, \cdots, \frac{1}{\varphi\left(q_{N}\right)}\right)
\end{aligned}
$$

where $\left(X_{1}, X_{2}, \cdots, X_{L}\right)$ is the transmitted symbols vector, $\operatorname{diag}(\cdot)$ means the diagonal matrix, $\varphi(\cdot)$ is defined as the Euler's totient function[16] and $\left\{q_{1}<q_{2}<\cdots<q_{N}\right\}$. To ensure that the modulated symbols vector can be recovered from the transmitted symbols vector accurately, $L$ must satisfy[8]

$$
L=\operatorname{LCM}\left(q_{1}, q_{2}, \cdots, q_{N}\right)
$$

where the LCM means the least common multiplier operation.

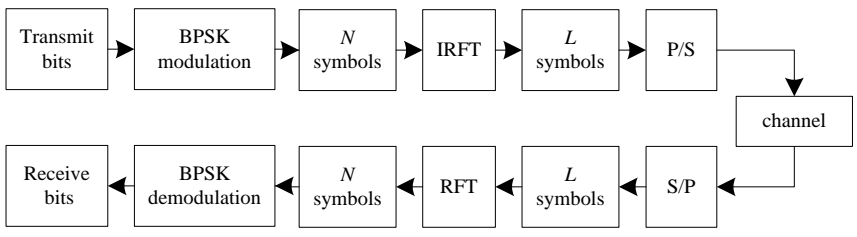

Figure 1: Diagram of RFMT system

The diagram of RFMT system is depicted in Fig. 1, where $\mathrm{P} / \mathrm{S}$ means the parallel to serial operation and S/P means the serial to parallel operation. In the transmitter, a group of transmitted bits are converted to a modulated vector $\mathbf{x}$ of $N$ symbols by BPSK modulation. Then, a transmitted vector of $L$ symbols is generated from $\mathbf{x}$ by the IRFT. In the receiver, the transmitted bits can be recovered by the RFT and BPSK demodulation.

The transmission efficiency of RFMT is defined as $\eta=N / L$, $\eta \in(0,1]$. The higher the value $\eta$, the better transmission efficiency. For example, if $\mathbf{R}_{\mathrm{s}}$ is $\left[\mathbf{c}_{1}, \mathbf{c}_{2}, \mathbf{c}_{4}, \mathbf{c}_{8}\right]^{\mathrm{T}}$, it means $N=4$ and $L=8$ according to (7). Then $\eta=N / L=4 / 8=0.5$. However, $L$ is very large and results in low efficiency if some transform vectors are used. For example, if $\mathbf{R}_{\mathrm{s}}$ is $\left[\mathbf{c}_{2}, \mathbf{c}_{4}, \mathbf{c}_{7}, \mathbf{c}_{10}\right]^{\mathrm{T}}$, $N=4$ but $L=140$. Thus, $\eta=4 / 140=0.029$.

\section{TLT-RFMT system}

In fact, Equation (7) is a sufficient condition for exact recovery of the transmitted symbols, including their signs and magnitudes. However, signs are sufficient to recover the transmitted bits. According the following theorem, we demonstrate that the recovery of signs of the received symbols requires much shorter transform vector in the proposed TLT-RFMT system. Thus, it is possible to significantly improve the transmission efficiency of RFMT by truncating the transform vector.

Before we present the following theorem, we would like to define $\mathbf{x}^{\prime}=\left(x_{1}^{\prime}, x_{2}^{\prime}, \cdots, x_{N}^{\prime}\right)$ as the received symbols vector after RFT, $\mathbf{R}_{\mathrm{st}}$ as the truncated matrix of $\mathbf{R}_{\mathrm{s}}$ and $L_{\mathrm{T}}$ as the transform vector length. Then the IRFT and RFT with $\mathbf{R}_{\text {st }}$ can be written

$$
\left(X_{1}, X_{2}, \cdots, X_{L_{\mathrm{T}}}\right)=\mathbf{x} \cdot \mathbf{R}_{\mathrm{st}}
$$

$$
\begin{aligned}
& \mathbf{x}^{\prime}= \\
& \frac{1}{L_{\mathrm{T}}}\left(X_{1}, X_{2}, \cdots, X_{L_{\mathrm{T}}}\right) \mathbf{R}_{\mathrm{st}}^{\mathrm{T}} \operatorname{diag}\left(\frac{1}{\varphi\left(q_{1}\right)}, \frac{1}{\varphi\left(q_{2}\right)}, \cdots, \frac{1}{\varphi\left(q_{N}\right)}\right)
\end{aligned}
$$

The product of $\mathbf{R}_{\mathrm{st}}$ and its transpose is denoted by

$$
\mathbf{D}=\mathbf{R}_{\mathrm{st}} \mathbf{R}_{\mathrm{st}}^{\mathrm{T}}=\left[\begin{array}{cccc}
d_{11} & d_{12} & \cdots & d_{1 N} \\
d_{21} & d_{22} & \cdots & d_{2 N} \\
\vdots & \vdots & & \vdots \\
d_{N 1} & d_{N 2} & \cdots & d_{N N}
\end{array}\right]
$$

If the elements of the $\mathbf{R}_{\mathrm{st}}$ matrix satisfy Theorem 1, i.e. the $\mathbf{D}$ matrix is dominated by its diagonal elements, we can recover the transmitted bits correctly by the signs of received symbols.

\section{Theorem 1. If the elements of the matrix D satisfy}

$$
d_{k k}>\sum_{j \neq k}\left|d_{j k}\right|, 1 \leq j, k \leq N
$$

then the elements of $\mathrm{x}^{\prime}$ have the same signs as those of $\mathrm{x}$ at the same index.

Proof: Substituting (8) into (9), we have

$$
\mathbf{x}^{\prime}=\mathbf{x} \cdot \mathbf{D} \cdot \frac{1}{L_{\mathrm{T}}} \cdot \operatorname{diag}\left(\frac{1}{\varphi\left(q_{1}\right)}, \frac{1}{\varphi\left(q_{2}\right)}, \cdots, \frac{1}{\varphi\left(q_{N}\right)}\right)
$$

The every element of $\mathbf{x}^{\prime}$ is written

$$
x_{k}^{\prime}=\frac{1}{L_{\mathrm{T}}} \cdot \frac{1}{\varphi\left(q_{k}\right)} \cdot \sum_{j=1}^{N} d_{j k} x_{j}, 1 \leq k \leq N
$$

Since $L_{\mathrm{T}}$ and the value of $\varphi(\cdot)$ are positive constant, they only decide the magnitude of the elements of $\mathbf{x}^{\prime}$. Their signs are determined by

$$
\sum_{j=1}^{N} d_{j k} x_{j}, 1 \leq k \leq N
$$

Recall the definition of $\mathbf{x}$ in (2). If the diagonal elements dominant the matrix $\mathbf{D}$, i.e. $d_{k k}>\sum_{j \neq k}\left|d_{j k}\right|$, the elements of $\mathbf{x}^{\prime}$ have the same signs as those of $\mathbf{x}$ at the same index. Consequently, we have proved that the transmitted bits can be correctly recovered from the signs of the received symbols in the TLT-RFMT. In other words, the signs of the received symbols keep unchanged as long as the matrix $\mathbf{D}$ is dominated by its diagonal elements.

\section{Analysis and Simulation}

As analyzed in RFMT system, its efficiency is determined by the length of $\mathbf{c}_{t}$. The shorter the length, the better transmission efficiency. However, it has a lower bound for the length. As the period of $c_{q}(n)$ is $q$, according to Theorem 1 , the lower bound of $L_{\mathrm{T}}$ is $q_{N}$, the max sequence number of RS of transform matrix. For example, if the matrix is $\left[\mathbf{c}_{2}, \mathbf{c}_{4}, \mathbf{c}_{7}, \mathbf{c}_{10}\right]^{\mathrm{T}}$, $L$ is 140 and $L_{\mathrm{T}}$ cannot be less than 10 . The following simulations show that TLT-RFMT also has non-uniform spectrum distribution and similar anti-noise performance of RFMT.

Consider that $\mathbf{R}_{\mathrm{s}}$ is $\left[\mathbf{c}_{2}, \mathbf{c}_{4}, \mathbf{c}_{7}, \mathbf{c}_{10}\right]^{\mathrm{T}}$ in RFMT. According to (7), $L$ is 140 . In TLT-RFMT, we consider $L_{\mathrm{T}}$ are four different values with $3 / 4,1 / 2,1 / 4$ of $L$. The spectrum distributions for RFMT and TLT-RFMT with different $L_{\mathrm{T}}$ are 
respectively shown in Fig. 2 and Fig 3. The results show that TLT-RFMT realizes the non-uniform spectrum distribution and its distribution is similar as RFMT.

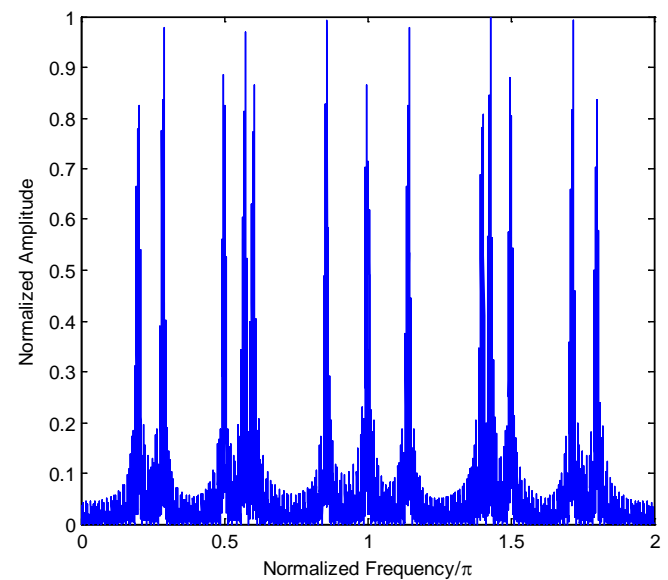

Figure 2: Spectrum Distribution for $\mathbf{R}_{\mathrm{s}}=\left[\mathbf{c}_{2}, \mathbf{c}_{4}, \mathbf{c}_{7}, \mathbf{c}_{10}\right]^{\mathrm{T}}$ with $L=140$

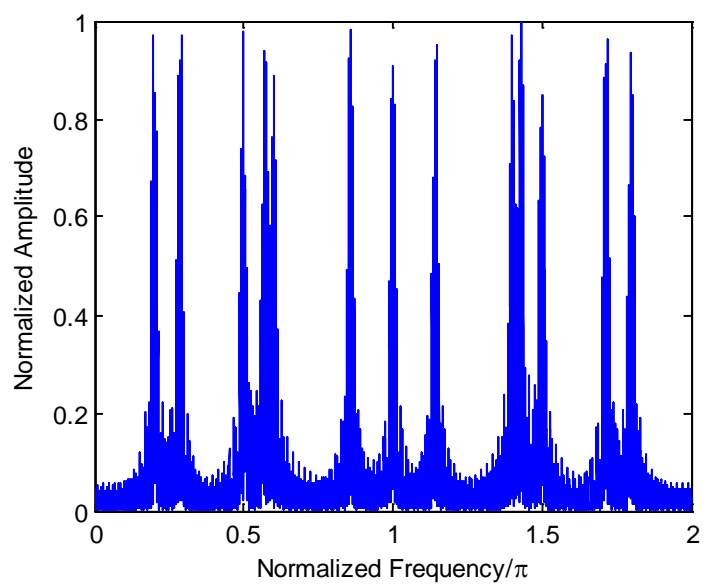

Figure 3-a: Spectrum Distribution for $\mathbf{R}_{\mathrm{s}}=\left[\mathbf{c}_{2}, \mathbf{c}_{4}, \mathbf{c}_{7}, \mathbf{c}_{10}\right]^{\mathrm{T}}$ with $L_{\mathrm{T}}=3 L / 4=105$

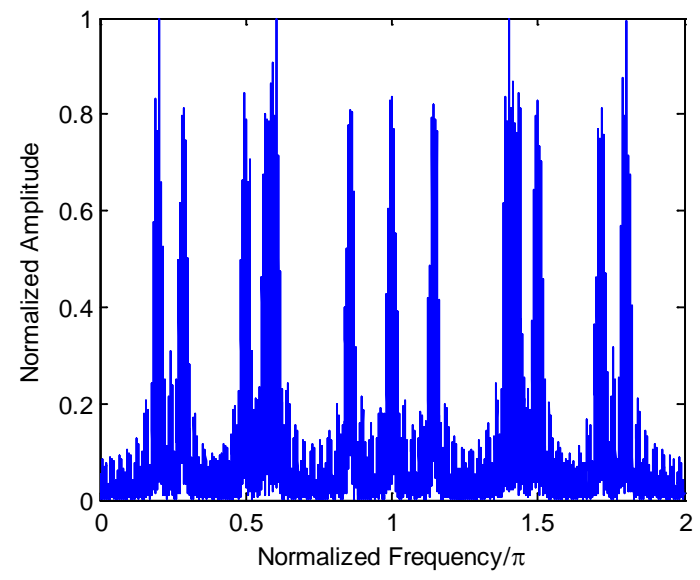

Figure 3-a: Spectrum Distribution for $\mathbf{R}_{\mathrm{s}}=\left[\mathbf{c}_{2}, \mathbf{c}_{4}, \mathbf{c}_{7}, \mathbf{c}_{10}\right]^{\mathrm{T}}$ with $L_{\mathrm{T}}=L / 2=70$

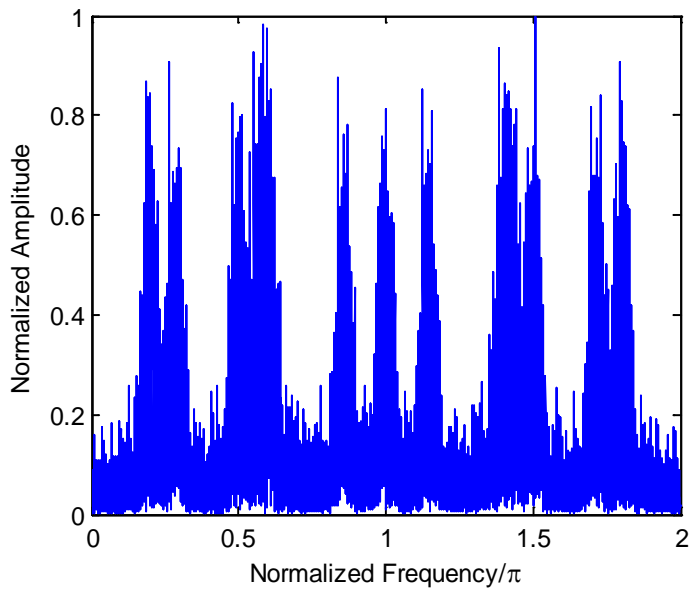

Figure 3-a: Spectrum Distribution for $\mathbf{R}_{\mathrm{s}}=\left[\mathbf{c}_{2}, \mathbf{c}_{4}, \mathbf{c}_{7}, \mathbf{c}_{10}\right]^{\mathrm{T}}$ with $L_{\mathrm{T}}=L / 4=35$

The anti-noise performance of TLT-RFMT system through simulations is evaluated over Additive White Gaussian Noise(AWGN) channel. Figure 4 shows the bit error ratio(BER) curves for two different methods with the transform matrix $\mathbf{R}_{\mathrm{s}}=\left[\mathbf{c}_{2}, \mathbf{c}_{4}, \mathbf{c}_{7}, \mathbf{c}_{10}\right]^{\mathrm{T}}$. TABLE I lists the transmission efficiency for different transform length. As shown in fig. 4, TLT-RFMT with $L_{\mathrm{T}}=105$ and 70 almost have the same anti-noise performances as RFMT. In other words, it also proves that the degradation of the spectrum distribution does not degrade the anti-noise performance when $L_{\mathrm{T}}$ is greater than $L / 2$, one half of the complete transform length. In addition, TABLE I shows that the transmission efficiency of the TLT-RFMT with $L_{\mathrm{T}}=70$ is 2 times of that of RFMT. If we use more aggressive truncation, $L_{\mathrm{T}}=35$, a quarter of $L$, Theorem 1 is still satisfied. Thus, TLT-RFMT is able to recover the transmitted bits. There is a performance gap within $2 \mathrm{~dB}$ between RFMT and TLT-RFMT when BER is about $10^{-5}$. However, the transmission efficiency of TLTRFMT is 4 times of that of RFMT. In other words, when the channel is better, TLT-RFMT is able to use shorter transform length for higher transmission efficiency; when the channel is worse, it is able to use longer length for better anti-noise performance.

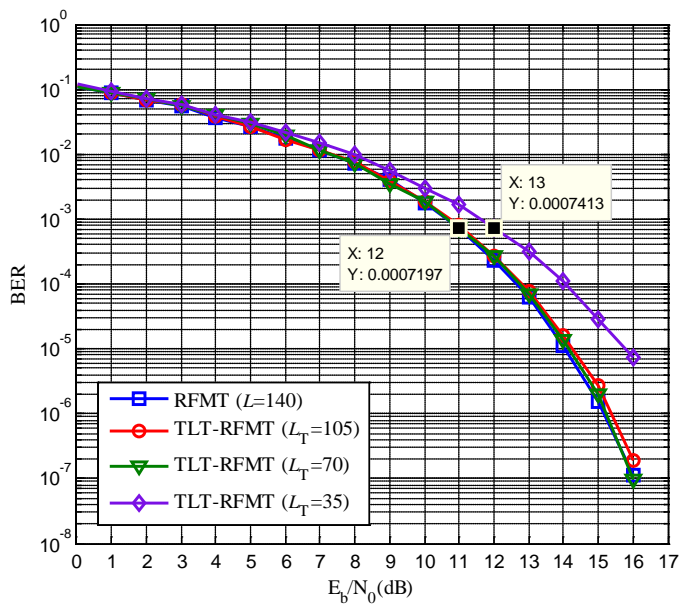

Figure 4: Anti-noise Performance for Different Methods 
TABLE I: Transmission Efficiency for Different Methods with $\mathbf{R}_{\mathrm{s}}=\left[\mathbf{c}_{2}, \mathbf{c}_{4}, \mathbf{c}_{7}, \mathbf{c}_{10}\right]^{\mathrm{T}}$

\begin{tabular}{|c|c|}
\hline Method & Transmission efficiency $(\eta)$ \\
\hline $\begin{array}{c}\text { RFMT } \\
(\boldsymbol{L}=\mathbf{1 4 0})\end{array}$ & $\mathbf{0 . 0 2 8}$ \\
\hline $\begin{array}{c}\text { TLT-RFMT } \\
\left(\boldsymbol{L}_{\mathbf{T}}=\mathbf{3 L} / \mathbf{4}=\mathbf{1 0 5}\right)\end{array}$ & 0.038 \\
\hline $\begin{array}{c}\text { TLT-RFMT } \\
\left(\boldsymbol{L}_{\mathbf{T}}=\mathbf{L} / \mathbf{2}=\mathbf{7 0}\right)\end{array}$ & $\mathbf{0 . 0 5 7}$ \\
\hline $\begin{array}{c}\text { TLT-RFMT } \\
\left(\boldsymbol{L}_{\mathbf{T}}=\mathbf{L} / \mathbf{4}=\mathbf{3 5}\right)\end{array}$ & 0.114 \\
\hline
\end{tabular}

\section{Conclusion}

The proposed TLT-RFMT system improves the transmission efficiency by truncating the transform vector. Analysis and simulation results show that the TLT-RFMT has non-uniform spectrum distribution. Moreover, its anti-noise performance is almost the same as the RFMT when the truncation is moderate. These results in the paper validate the practical feasibility of TLT-RFMT non-uniform scheme.

\section{Acknowledgements}

This work was supported by National Natural Science Foundation of China under Grants 61471022, and NSAF under Grant U1530117.

\section{References}

[1] Bingham JA. "Multicarrier modulation for data transmission: An idea whose time has come", IEEE Communications Magazine, 28(5), pp. 5-14, (1990)

[2] Nee Rv, Prasad R. "OFDM for wireless multimedia communications”, Artech House(2000)

[3] Nikookar H, Prasad R. "Multicarrier transmission with nonuniform carriers in a multipath channel", IEEE International Conference on Universal Personal Communications, 2, pp. 628-632, (1996)

[4] Roque D, Siclet C, Siohan P. “A performance comparison of FBMC modulation schemes with short perfect reconstruction filters”, 19th IEEE International Conference on Telecommunications(ICT), pp. 1-6, (2012)

[5] Xie S, Chen F, Kwong S, Ji F. "Generalized Multicarrier Modulation with Pseudo Nonuniform Carrier Spaces”, IEEE International Conference on Networks Security, Wireless Communications and Trusted Computing(NSWCTC), pp. 196199, (2009)

[6] Vaidyanathan PP. "Ramanujan Sums in the Context of Signal Processing-Part I: Fundamentals”, IEEE Transactions on Signal Processing, 62, (16), pp. 4145-4157, (2014)

[7] Samadi S, Ahmad MO, Swamy M. "Ramanujan sums and discrete Fourier transforms”, IEEE Signal Processing Letters, 12(4), pp. 293-296, (2005)

[8] Lina Zhou, Zulin Wang, Jiadong Shang. "Novel nonuniform multi-tone system based on Ramanujan sums”,
Journal of Beijing University of Aeronautics and Astronautics, 40, (3), pp. 338-343, (2014)

[9] Zhou LN, Shang JD, Yang L. "Research on RamanujanFMT modulation and the efficient implementation algorithm", Applied Mechanics and Materials, 380, pp. 1693-1696, (2013) [10] Kumar GS, Sandeepkumar V, Anuradha S. "Peak-toaverage power ratio analysis for NC-OQAM/OFDM transmissions”, IEEE International Conference on Microelectronics, Computing and Communication, pp. 1-5, (2016)

[11] Rajbanshi R, Wyglinski AM, Minden GJ. “An efficient implementation of NC-OFDM transceivers for cognitive radios”, 1st IEEE International Conference on Cognitive Radio Oriented Wireless Networks and Communications, pp. 1-5, (2006)

[12] Zhang G, Feng S. "Subcarrier allocation algorithms based on graph-coloring in Cognitive Radio NC-OFDM system”, 3rd IEEE International Conference on Computer Science and Information Technology (ICCSIT), pp. 535-540, (2010)

[13] Xie S, Chen F, Kwong S, Ji F. "Generalized Multicarrier Modulation with Pseudo Nonuniform Carrier Spaces”, IEEE International Conference on Networks Security, Wireless Communications and Trusted Computing, pp. 196-199, (2009) [14] Franz S, Mitra SK, Schmidt J, Doblinger G. "Warped discrete Fourier transform: a new concept in digital signal processing”, IEEE International Conference on Acoustics, Speech, and Signal Processing, (2002)

[15] Sugavaneswaran L, Xie S, Umapathy K, Krishnan S. “Time-frequency analysis via Ramanujan sums”, IEEE Signal Processing Letters, 19, (6), pp. 352-355, (2012)

[16] Apostol, Tom M. "Introduction to analytic number theory”, Springer Science \& Business Media(2013) 